\title{
Ezrin is required for epithelial-mesenchymal transition induced by TGF- $\beta 1$ in A549 cells
}

\author{
MIAO-JUAN CHEN ${ }^{*}$, XUE-JUAN GAO* , LI-NA XU, TENG-FEI LIU, XIAO-HUI LIU and LANG-XIA LIU \\ Key Laboratory of Functional Protein Research of Guangdong Higher Education Institutes, \\ Institute of Life and Health Engineering, Jinan University, Guangzhou 510632, P.R. China
}

Received March 23, 2014; Accepted July 2, 2014

DOI: $10.3892 /$ ijo.2014.2554

\begin{abstract}
Epithelial mesenchymal transition (EMT) has been shown to play a role in cellular differentiation during development and tumor invasion. However, the precise molecular mechanisms of EMT are not fully elucidated. Previous studies suggested that the mechanism underlying the possible involvement of ezrin in EMT process might be different from that of moesin, another ERM protein. In our study, we examined the role of ezrin in actin filament reorganization and cell metastasis during TGF- $\beta 1$-induced alveolar EMT. Suppressing ezrin expression limited morphological changes and actin filament remodeling, decreased cell migration and invasion during EMT. Immunofluorescence experiments indicated that EMT characteristics in lung cancer cells are associated to differential ezrin subcellular localization. We also found that podocalyxin interacted with ezrin after TGF- $\beta 1$ induction. Therefore, ezrin is an important regulator of the EMT process, and its function might possibly be mediated by the ezrin-podocalyxin interaction during TGF- $\beta 1$-induced alveolar EMT. Our finding provides important new insights into the mechanisms of action of the ERM proteins in the TGF- $\beta 1$-induced alveolar EMT.
\end{abstract}

\section{Introduction}

Epithelial mesenchymal transition (EMT) not only plays crucial roles in embryonic development or tissue repair, but is also involved in fibrotic diseases and cancer progression (1-3). During EMT, cells undergo profound phenotypic changes including the loss of cell-cell adhesion, the loss of cell polarity, the reorganization of cytoskeleton, and the acquisition of migratory and invasive properties $(4,5)$. A network of transcriptional

Correspondence to: Professor Lang-Xia Liu, Key Laboratory of Functional Protein Research of Guangdong Higher Education Institutes, Institute of Life and Health Engineering, Jinan University, Guangzhou 510632, P.R. China

E-mail: langxialiu@gmail.com; tliulx@jnu.edu.cn

*Contributed equally

Key words: ezrin, epithelial-mesenchymal transition, actin filament reorganization, cell metastasis, podocalyxin regulators is involved in the control of EMT, which is coupled to posttranscriptional and posttranslational modifications that amplify the initial signals (6). Several transcription factors have been shown to be involved in this process, such as the Snail family of zinc-finger transcription factors and the basic helix-loop-helix factors Twist $(7,8)$. These transcription factors downregulate epithelial markers (E-cadherin) and upregulate mesenchymal markers (fibronectin, vimentin), which induce EMT and consequently promote the development of metastatic properties $(9,10)$. Many extracellular matrix components and cytokines, including Wnt, hepatocyte growth factor, epidermal growth factor (EGF) and transforming growth factor- $\beta$ (TGF- $\beta$ ), can elicit EMT. Among them, TGF- $\beta$ is a powerful inducer of EMT (11). Depending on the specific cellular context, different signaling pathways during TGF- $\beta$-induced EMT can be activated and contribute to establish an organizing center that in turn controls morphogenetic movement and specification (12).

Idiopathic pulmonary fibrosis (IPF) is a specific form of chronic progressive fibrosing interstitial pneumonia of unknown cause that is limited to the lungs (13). A major factor in IPF pathogenesis is thought to be an aberrant activation of alveolar epithelial cells (AECs). AECs can transformed into (myo) fibroblasts, which secrete an excessive amount of collagen to form fibers, impairing organ function (14). There is increasing evidence that EMT is involved in these processes during pulmonary fibrogenesis and TGF- $\beta$ is a major inducer of EMT in the lungs (2). Previous studies indicated the alveolar epithelial cells (AEC) underwent EMT during the pulmonary fibrosis induced by TGF- $\beta$, and TGF- $\beta 1$ induced A549 AEC to undergo EMT via Smad2 activation $(15,16)$. These results suggest that AEC serve as a source of fibroblasts in lung fibrosis and highlight the potentially critical role of EMT in the induction of fibrosis in the lung. Core of this process is cytoskeleton reorganization (5). After induction by proinflammatory cytokines, actin filament architecture changes from cortical actin to stress fibers. However, the precise mechanism underlying these structural rearrangements remains unknown.

Ezrin, radixin and moesin, known as the ERM proteins, are a group of membrane-cytoskeleton linkers, which are closely associated with actin cytoskeleton remodeling $(17,18)$. Two groups have shown the role of ERM proteins in the actin filaments rearrangement during EMT $(19,20)$. ERM proteins may be involved in regulating alveolar structure and lung homeostasis. Moreover, dysregulation of the ERM-RAGE 
complex might be an important step in rearrangement of the actin cytoskeleton during proinflammatory cytokine-induced EMT of human alveolar epithelial cells. Recent study showed that increased moesin expression promotes EMT by regulating actin filament remodeling, but ezrin expression decreased in NMuMG cells or remained unchanged in A549 cells during TGF- $\beta$-induced EMT, suggesting that the regulating mechanism of EMT by ezrin may be different from that by moesin if it does play a role in this process (21).

Podocalyxin (PODXL), as a member of the CD34 family, is a type I transmembrane glycoprotein, playing an important role in regulating cell adhesion and cell morphology (22). It has a number of interacting partners, including the actin binding protein ezrin, the adhesion molecule L-selectin and $\mathrm{Na}^{+} / \mathrm{H}^{+}$ exchanger regulatory factor (NHERF) $(23,24)$. PODXL could increase migration and invasion, MMP expression, and activation of MAPK and PI3K activity in MCF7 and PC3 cells through interaction with ezrin (25). Moreover, PODXL is markedly increased and required for TGF- $\beta$ induced EMT of A549 cells (26). During this process, PODXL interacts with collagen type I, which may control cell migration by regulating the dynamics of cell protrusion formation.

In our study, we examine the role of ezrin in actin filament reorganization and cell metastasis during TGF- $\beta 1$-induced alveolar EMT. Our finding also revealed an association between ezrin and PODXL during EMT, suggesting ezrinpodocalyxin complex might be important in actin filament remodeling during TGF- $\beta 1$-induced alveolar EMT.

\section{Materials and methods}

Antibodies. Monoclonal ezrin antibody, monoclonal or polyclonal podocalyxin antibodies and monoclonal vimentin antibody were obtained from Santa Cruz Biotechnology. Monoclonal E-cadherin antibody was purchased from BD (Becton-Dickinson Co.).PolyclonalGAPDH antibody was from Cell Signaling Technologies (Beverly, MA, USA). Monoclonal $\beta$-actin antibody was from Proteintech. Secondary antibodies conjugated to Alexa Fluor 488 or Alexa Fluor 594 was from Beyotime Institute of Biotechnology (China). Secondary antibodies conjugated to peroxidase were obtained from Jackson ImmunoResearch Laboratories Inc. (West Grove, PA, USA).

Cell culture and treatment. Human bronchoalveolar carcinoma cell H358, human lung adenocarcinoma cell A549 and human lung adenocarcinoma cell H1299 were purchased from the American Type Culture Collection (ATCC, USA). All cell lines were grown in high-glucose Dulbecco's modified Eagle's medium (DMEM) supplemented with $10 \%$ fetal bovine serum (FBS) and maintained at $37^{\circ} \mathrm{C}$ with $5 \% \mathrm{CO}_{2}$. A549 cells were treated with $5 \mathrm{ng} / \mathrm{ml}$ recombinant human TGF- $\beta 1$ (Pepro Tech) for 24 or $48 \mathrm{~h}$ to induce EMT.

RNA interference. Sequences of ezrin siRNA were designed and synthesized by Thermo Scientific Dharmacon. Ezrin siRNA contains 4 individual siRNAs, Ezrin-siRNA-1: 5'-GCU CAA AGA UAA UGC UAU GTT-3' (sense) and 5'-CAU AGC AUU AUC UUU GAG CTT-3' (antisense). Ezrin-siRNA-2: 5'-GGA AUC AAC UAU UUC GAG ATT-3' (sense) and 5'-UCU CGA AAU AGU UGA UUC CTT-3' (antisense).
Ezrin-siRNA-3: 5'-GCG CAA GGA GGA UGA AGU UTT-3' (sense) and 5'-AAC UUC AUC CUC CUU GCG CTT-3' (antisense). Ezrin-siRNA-4: 5'-GCG CGG AGC UGU CUA GUG ATT-3' (sense) and 5'-UCA CUA GAC AGC UCC GCG CTT-3' (antisense). The negative control siRNA was also purchased from Thermo Scientific Dharmacon. The cells were transfected with ezrin siRNA or negative control siRNA using Lipofectamine 2000 (Invitrogen, Carlsbad, CA, USA) according to the manufacturer's instructions.

Immunofluorescent analysis. Cells were fixed with $4 \%$ paraformaldehyde for $30 \mathrm{~min}$, permeated with $0.1 \%$ Triton X-100 for $10 \mathrm{~min}$ at room tempreture (RT), followed by blocking in $10 \%$ normal goat serum for $1 \mathrm{~h}$, and then incubated with the primary antibody at $4^{\circ} \mathrm{C}$ overnight. After washing, the slides were incubated with Alexa flour 488-conjugated secondary antibody, followed by nuclear counterstaining with DAPI for 10 min. F-actin was stained using rhodamine conjugatedphalloidin (Invitrogen). Cells were imaged using a 40X EC Plan Neofluar/1.30 oil immersion objective on an inverted laser-scanning confocal microscope (LSM510 META, Carl Zeiss) and images were captured using Zeiss software.

Coimmunoprecipitation. Cells were rinsed with PBS twice and lysed in lysis buffer (20 mM Tris, pH 7.5, $150 \mathrm{mM} \mathrm{NaCl}$, $1 \%$ Triton $\mathrm{X}-100)$ with $1 \mathrm{X}$ proteinase inhibitor cocktail (Roche), $1 \mathrm{mM}$ sodium fluoride, and $1 \mathrm{mM}$ sodium orthovanadate (Amersco) for $30 \mathrm{~min}$ at $4^{\circ} \mathrm{C}$. The lysates were clarified by centrifugation at $13200 \mathrm{rpm}$ for $20 \mathrm{~min}$. Protein concentrations were detected using the BCA protein assay kit. After pre-clear, $1.5 \mathrm{mg}$ total protein was immunoprecipitated with the indicated primary antibody overnight, and incubated with $20 \mu \mathrm{l}$ Protein A/G Plus-Agarose beads for $4 \mathrm{~h}$ at $4^{\circ} \mathrm{C}$. The immunoprecipitates and the lysates were subjected to western blotting using the antibody indicated.

Wetstern blotting. Cells were grown to confluence on culture dishes, and lysed in ice-cold cell lysis buffer. Lysates were centrifuged at $13,200 \mathrm{rpm}$ for $20 \mathrm{~min}$ at $4^{\circ} \mathrm{C}$ to obtain the proteins, and then protein concentration was determined by the BCA protein assay. The protein lysates were separated by SDS-PAGE and then transferred to PVDF membrane (Millipore). The membranes were blocked with 5\% non-fat milk solution for $1 \mathrm{~h}$ at room temperature and incubated in primary antibody dissolved in block solution at $4^{\circ} \mathrm{C}$ overnight. The proteins were probed by antibody against ezrin, E-cadherin, vimentin or $\beta$-actin. After washing, the membrane was incubated with horseradish peroxidase-conjugated secondary antibody corresponding to the primary antibody for $1 \mathrm{~h}$ at room temperature, and visualized using ECL.

Would-healing assay. Adhered cell monolayers were scratched with a $200-\mu 1$ pipette tip and grown in DMEM medium with $10 \% \mathrm{FBS}$ at $37^{\circ} \mathrm{C}$ with $5 \% \mathrm{CO}_{2}$. Wound healing capacity was monitored by microscopy at 0,12 and $24 \mathrm{~h}$.

Cell invasion assay. After siRNA transfection, TGF- $\beta 1$ induction was started $48 \mathrm{~h}$ before the assay. The inserts were precoated with $40 \mu \mathrm{l} \mathrm{BD}$ Matrigel and then cells $\left(5 \times 10^{4}\right)$ were seeded in the upper chamber and incubated for $12 \mathrm{~h}$. The cells 
A

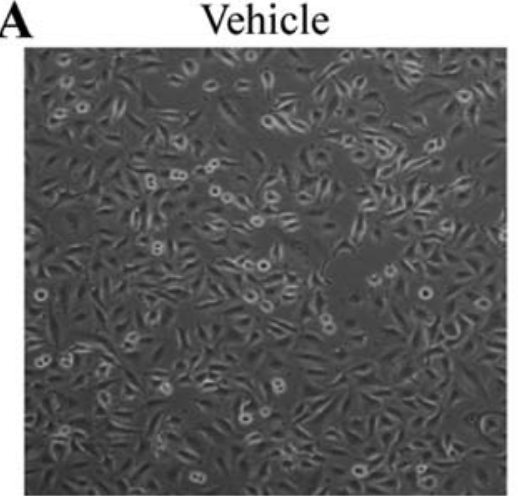

B
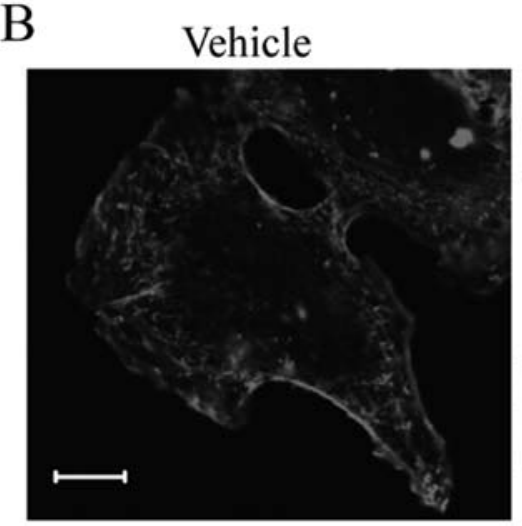

TGF- $\beta 148 \mathrm{~h}$

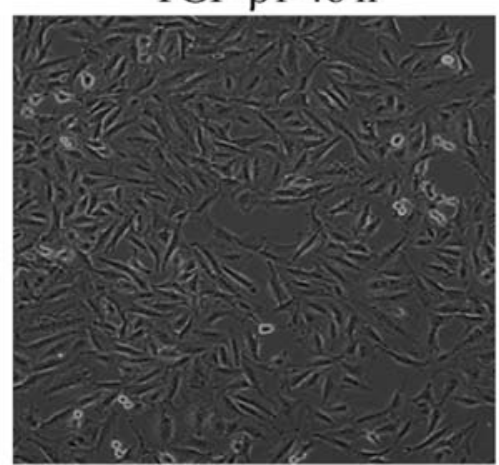

TGF- $\beta 148 \mathrm{~h}$
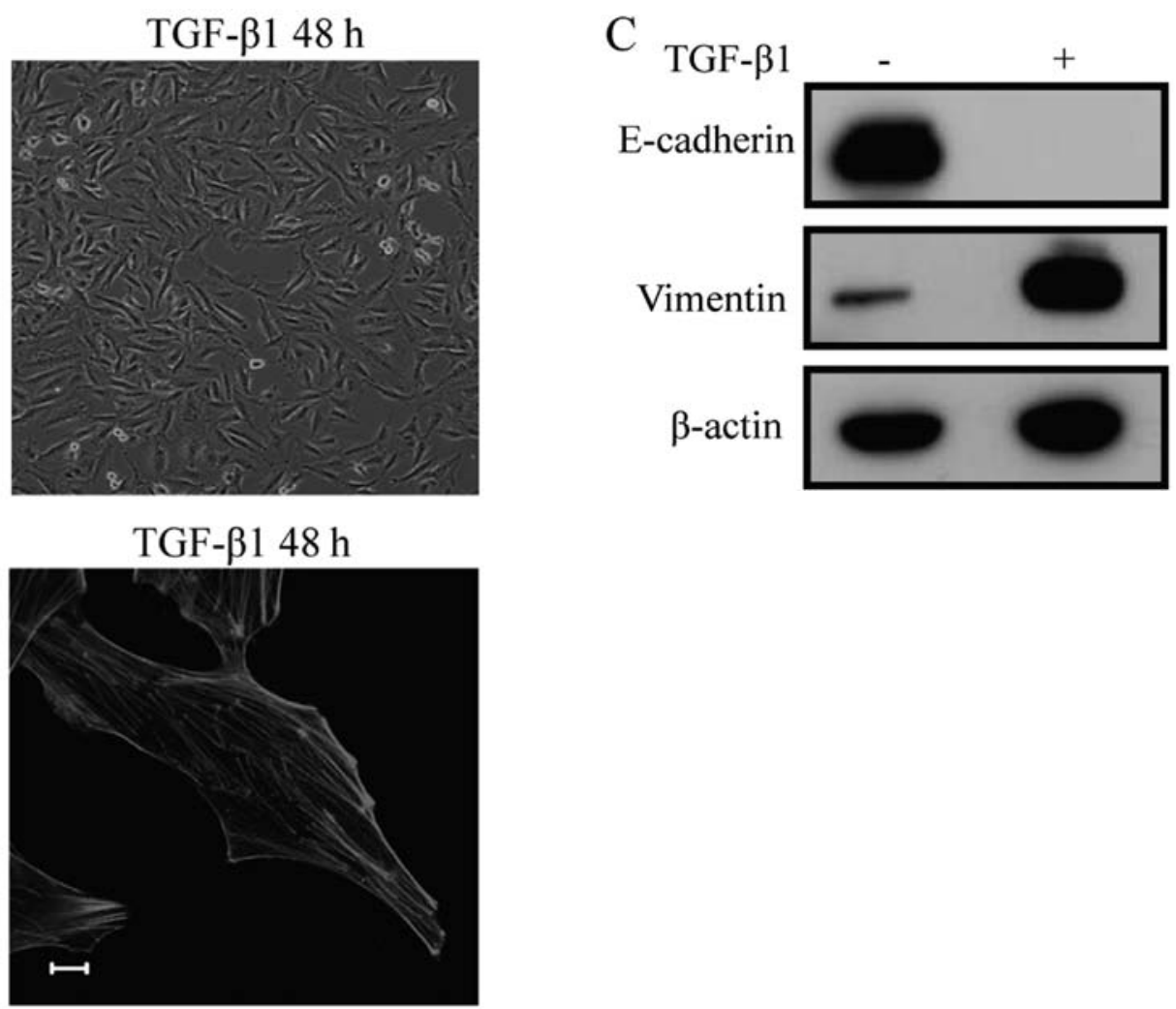

Figure 1. EMT was induced by TGF- $\beta 1$. (A and B) A549 cells were cultured in medium with or without 2 ng/ml TGF- $\beta 1$ for 48 h. Cellular morphology was photographed by phase contrast microscope (A), and actin cytoskeleton was visualized by rhodamine conjugated-phalloidin staining and photographed by confocal microscope (B). Scale bar, $10 \mu \mathrm{m}$. (C) The effect of TGF- $\beta 1$ on expression of EMT markers, E-cadherin and vimentin, were examined by western blotting. $\beta$-actin from the same loading was used as control.

were fixed and stained with crystal violet. Migrated cells in 5 randomly chosen fields of each well were counted.

\section{Results}

Suppressing ezrin expression limits morphological changes and actin filament remodeling during EMT. The morphological and biochemical features of A549 cells after TGF- $\beta 1$ treatment were examined to confirm that EMT properties were induced under our culture conditions. After TGF- $\beta 1$ treatment, A549 cells underwent phenotypic changes including the acquisition of spindle shape, the reorganization of actin filaments from cortical thin bundles to thick parallel bundles or actin stress fibers. In addition, the loss of expression of the epithelial marker, E-cadherin, and an increase of expression of the mesenchymal marker, vimentin further confirmed that EMT properties were induced under our culture conditions (Fig. 1). To examine the functional significance of ezrin during EMT, we chose to knock down ezrin expression by using siRNA. Two days following the transfection with ezrin siRNA, the level of ezrin expression was reduced by $\sim 70 \%$ as compared with the cells transfected with control siRNA (Fig. 2A and B). Additionally, there was no significant difference in $\mathrm{LDH}$ release in ezrin siRNA transfected cells as compared with the control, suggesting ezrin siRNA has no cytotoxicity on the cells tested (Fig. 2C). After TGF- $\beta 1$ treatment, control cells expressing negative control siRNA showed decreased expression of E-cadherin and increased expression of vimentin during EMT. Cells transiently transfected with ezrin siRNA exhibited increased expression of E-cadherin and decreased expression of vimentin in contrast to negative control siRNA cells (Fig. 3A).

Compared with negative control siRNA cells, ezrin siRNA cells treated with TGF- $\beta$ had different morphology and actin filament remodeling. After TGF- $\beta 1$ treatment, actin filaments in negative control siRNA cells changed from a cortical actin network to thick bundles, or actin stress fibers and the cells elongated and became spindle shape. However, cells transfected with ezrin siRNA had fewer and thinner actin filaments, accompanied by incomplete morphological transition as compared with control siRNA cells (Fig. 3B). These results indicate that ezrin is associated with morphological changes and actin filament remodeling during EMT.

Suppressing ezrin expression during EMT decreases cell migration and invasion. The acquisition of migratory and invasive properties is one of the phenotypic changes during EMT. To determine the relationship between ezrin and cell migratory and invasive abilities during EMT, we used ezrin siRNA to suppress ezrin expression and observed cell migration and invasion after TGF- $\beta 1$ treatment. In a wound-healing assay, cells transfected with ezrin siRNA showed decreased wound healing at the indicated time as compared with control siRNA cells (Fig. 4A). Moreover, the invasive potential of cells transfected with ezrin siRNA decreased as compared with control siRNA cells (Fig. 4B and C). These results demon- 


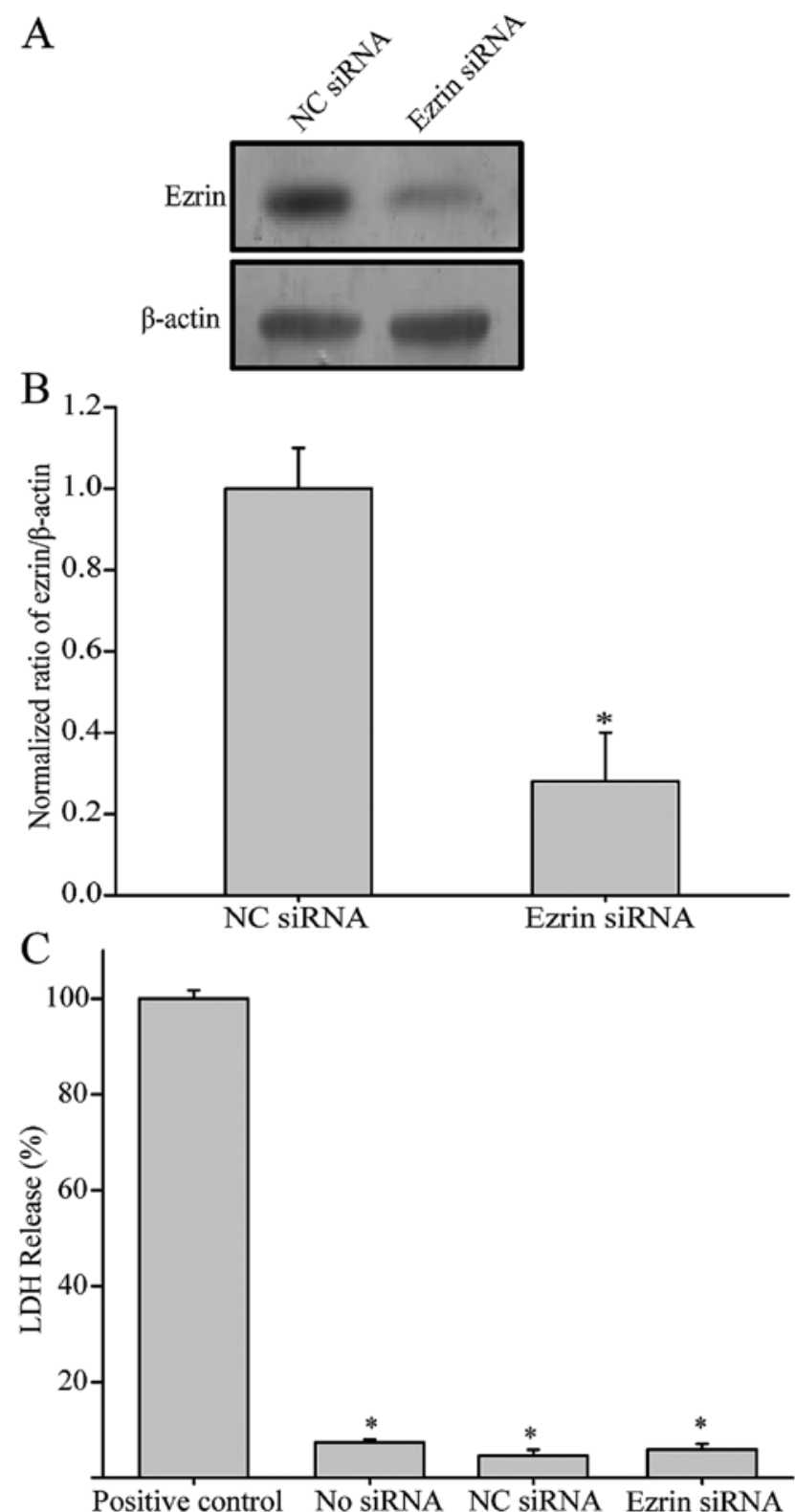

Figure 2. The expression of ezrin is suppressed with specific ezrin siRNA. (A and B) A549 cells were transfected with ezrin siRNA or negative control siRNA (NC siRNA). After transfection for $48 \mathrm{~h}$, cells were collected and ezrin expression was revealed by western blotting. $\beta$-actin from the same loading was used as control. Data shown are means \pm SEM from three independent cell preparations, ${ }^{*} \mathrm{p}<0.05$, significant compared with the control group. (C) The cytotoxicity of ezrin siRNA was detected using LDH cytotoxicity assay kit. A549 cells were transfected with ezrin siRNA or NC siRNA. After transfection for $48 \mathrm{~h}$, the extracellular LDH in culture media from ezrin siRNA group or NC siRNA group was measured using LDH cytotoxicity assay kit. Data shown are means \pm SEM from three independent cell preparations. ${ }^{*} \mathrm{p}<0.05$ compared with positive control.

strated that ezrin is involved in regulating cell migration and invasion during EMT, further confirming ezrin is required for TGF- $\beta 1$-induced EMT in A549 cells.

Differential ezrin localization is associated with EMT characteristics in lung cancer cells. To further assess the involvement of ezrin expression in TGF- $\beta 1$-induced EMT, we observed the change of ezrin expression level in A549 cells treated with TGF- $\beta$. However, the result showed that the expression level of ezrin has no change in A549 cells following TGF- $\beta$ treatment (Fig. 5A), which is consistent with the report from Haynes et al (21). Subsequently, we observed the change of ezrin expression level in different lung cancer cell lines (H358, A549 and H1299). Though these cell lines underwent EMT to different extent, there was no obvious difference in the levels of ezrin protein (Fig. 5B). However, we found that ezrin was differently distributed in these lung cell lines (Fig. 5C). In H358 cells, ezrin was localized at microvilli throughout the entire cell. In contrast, A549 cells showed that ezrin was distributed in the cytoplasm. In H1299 cells, ezrin was found in the cytoplasm, and a proportion of it was colocalized with F-actin. The localization of ezrin and its colocalization with F-actin were in accordance with the invasion ability of these cell lines, which is one of the EMT properties. Taken together, the results demonstrated that differential ezrin localization, rather than total ezrin protein levels, is associated with EMT characteristics in lung cell lines, suggesting ezrin may be involved in the mechanism of TGF- $\beta 1$-induced EMT through the change of its distribution.

The association of ezrin and podocalyxin increases during $E M T$. A recent study found that PODXL is involved in the EMT process through regulating the loss of epithelial features and acquisition of a motile phenotype. PODXL expression was induced upon TGF- $\beta 1$ treatment (26). We asked whether PODXL is associated with ezrin during TGF- $\beta 1$-induced EMT, and then used coimmunoprecipitation to detect the interaction between PODXL and ezrin. Our result showed that PODXL interacted with ezrin during TGF- $\beta 1$-induced EMT (Fig. 6). These results suggest that ezrin may be involved in regulating TGF- $\beta 1$-induced EMT through interaction with PODXL.

\section{Discussion}

A number of investigations into the molecular events of TGF- $\beta 1$-induced EMT have paved the way for the design of improved specific therapies. In the present study, we provide information regarding the role of ezrin in TGF- $\beta 1$-induced EMT, which may be associated with its localization and target proteins. These observations broaden knowledge of the precise molecular mechanism mediating TGF- $\beta 1$-induced EMT, which is important for developing strategies to inhibit or reverse EMT.

Ezrin, as a membrane-cytoskeleton linker, plays a privotal role in tumor invasion and metastasis (27-29). Ezrin can regulate the assembly of cytoskeleton elements to promote cytoskeletal reorganization and phenotypic alternation in cells, and facilitate cell migration and invasion $(30,31)$. Overexpression of ezrin has been shown to enhance metastatic potential in various types of tumors, while downregulation of ezrin reduced the expression of $\beta$-catenin but enhanced the expression of E-cadherin $(32,33)$. It has been found that ERM proteins are involved in regulating cytokine-induced EMT of human alveolar epithelial cells $(19,20)$. The expression of moesin increased, which promotes EMT by regulating actin filament remodeling. However, the expression of ezrin has no change during TGF- $\beta 1$-induced EMT (21). The role of ezrin in TGF- $\beta 1$-induced EMT remains unknown. In the present study, 


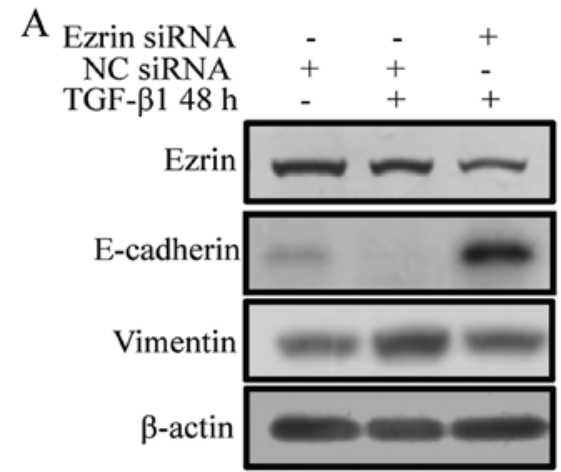

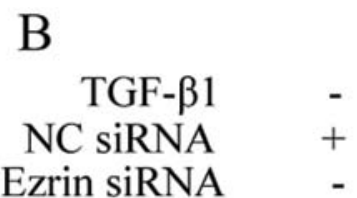

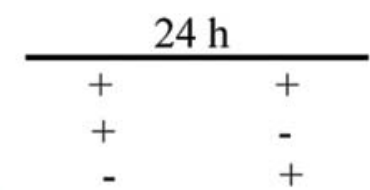

\section{DAPI}
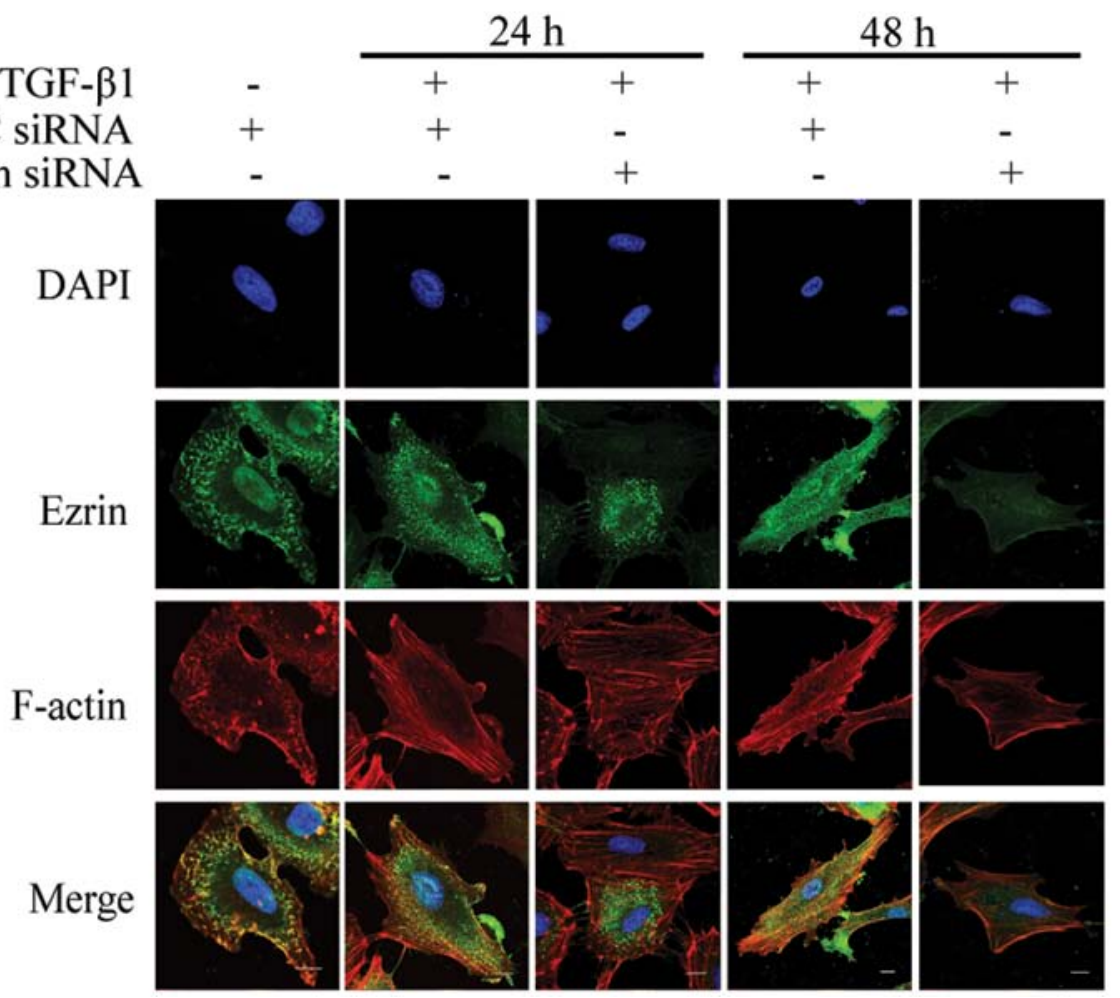

Figure 3. Suppressing ezrin expression limits morphological changes and actin filament remodeling during EMT. (A) The changes in protein expression during EMT are shown. NC siRNA, and ezrin siRNA cells were treated without or with TGF- $\beta 1$ for $48 \mathrm{~h}$. Blots were probed with antibodies for E-cadherin, vimentin, and ezrin. $\beta$-actin from the same loading was used as control. (B) The changes in cellular morphology and actin filament during EMT were detected. NC siRNA and ezrin siRNA cells were treated with TGF- $\beta 1$ for 24 or $48 \mathrm{~h}$. Fixed cells were incubated with anti-ezrin mAb (green) and rhodamine conjugatedphalloidin (red), and then photographed using a confocal microscope.

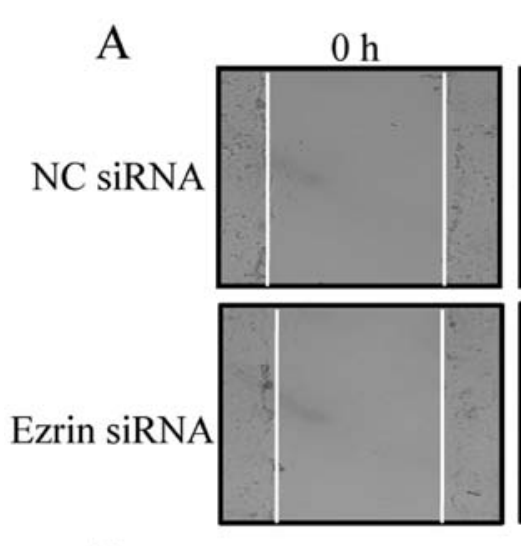

B

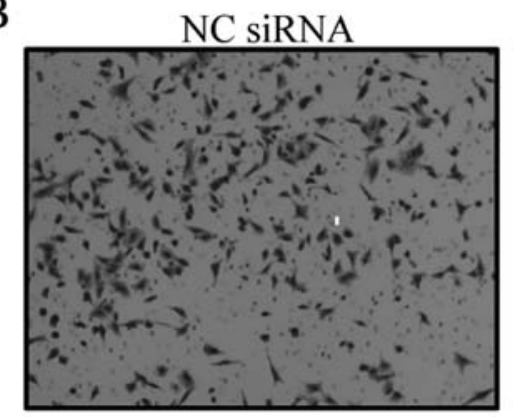

$12 \mathrm{~h}$
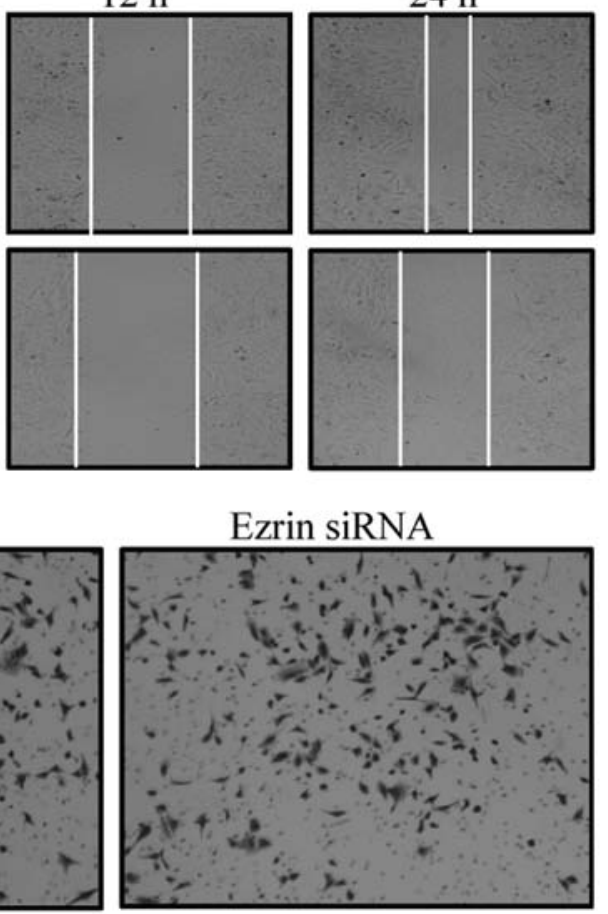

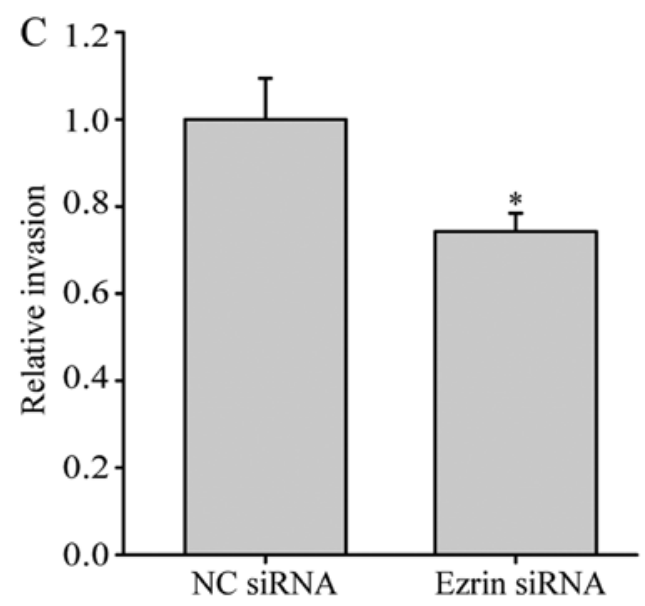

Figure 4. Suppression of ezrin expression during EMT decreases cell migration and invasion. (A) Ezrin siRNA knockdown decreases cell migration. NC siRNA and ezrin siRNA cells were treated with TGF- $\beta 1$ for $48 \mathrm{~h}$ and scratched with a $200-\mu 1$ pipette tip. Images were acquired by phase contrast microscope at the indicated time. (B and C) The cells transfected with NC siRNA or ezrin siRNA were seeded in the inserts precoated with BD Matrigel and incubated for $12 \mathrm{~h}$. The NC siRNA and ezrin siRNA cells were quantified after treated for $48 \mathrm{~h}$ with TGF- $\beta 1$. Data shown are means \pm SEM from three independent cell preparations. " $\mathrm{p}<0.05$, significant compared with the control group. 

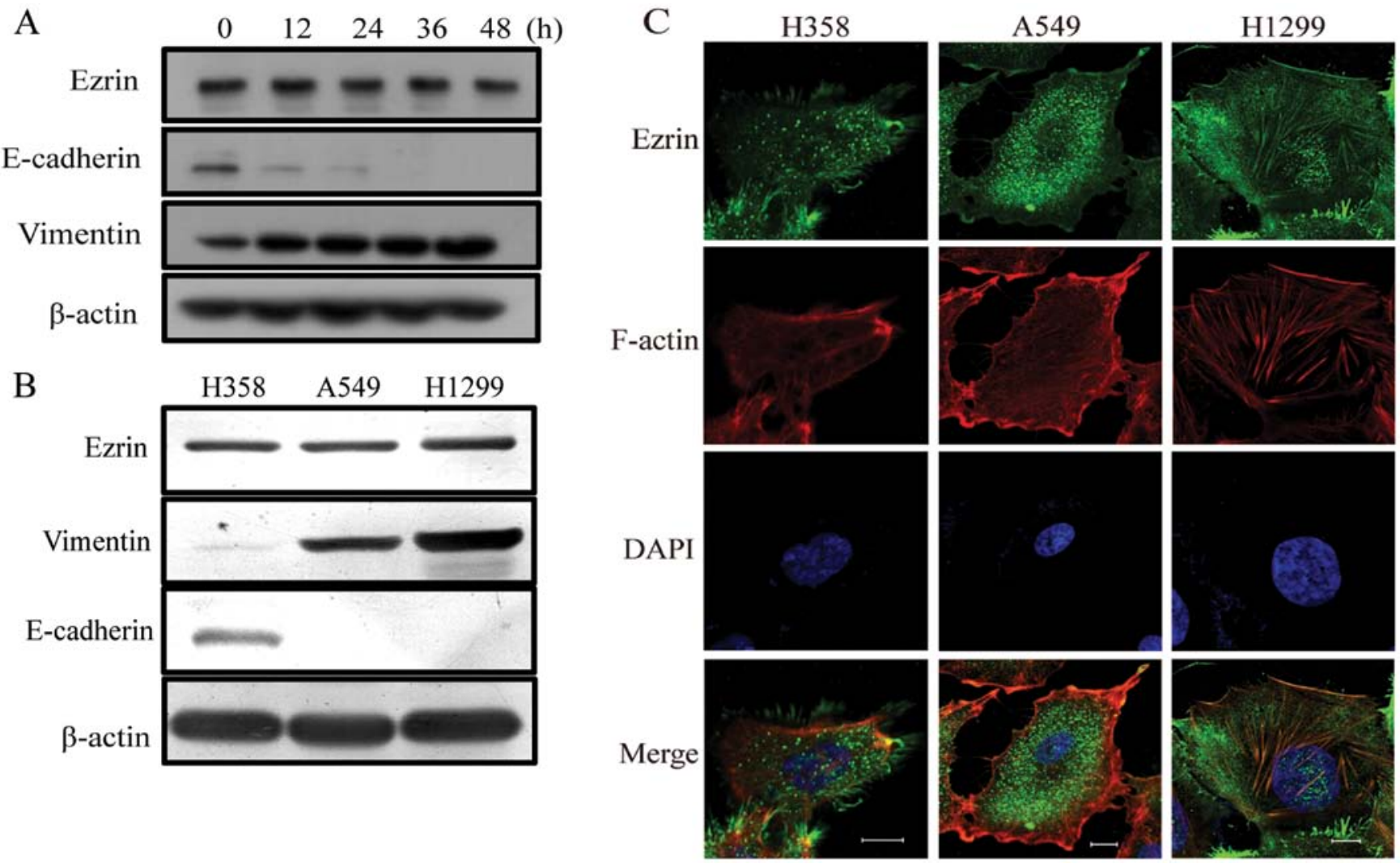

Figure 5. Differential ezrin localization is associated with EMT characteristics in lung cancer cells. (A) The expression level of ezrin has no change in A549 cells following TGF- $\beta$ treatment. A549 cells were treated with $2 \mathrm{ng} / \mathrm{ml}$ TGF- $\beta 1$ for the indicated time and the cells were collected for western blotting. (B) The level of ezrin protein has no clear difference among lung cell lines undergoing EMT to different extent. Ezrin expression in lung cell lines was revealed by western blotting. $\beta$-actin from the same loading was used as control. (C) Differential localization of ezrin in lung cell lines. Cells were incubated with anti-ezrin $\mathrm{mAb}$ (green) and rhodamine conjugated-phalloidin (red), followed by anti-mouse Alexa 488 secondary antibody. Nucleus was visualized using DAPI staining (blue). Ezrin-rich microvilli are grouped at the surface in H358 cells. In A549 cells, ezrin distributes in the cytoplasm. In H1299 cells, ezrin was found in the cytoplasm, and a proportion of it was colocalized with F-actin.

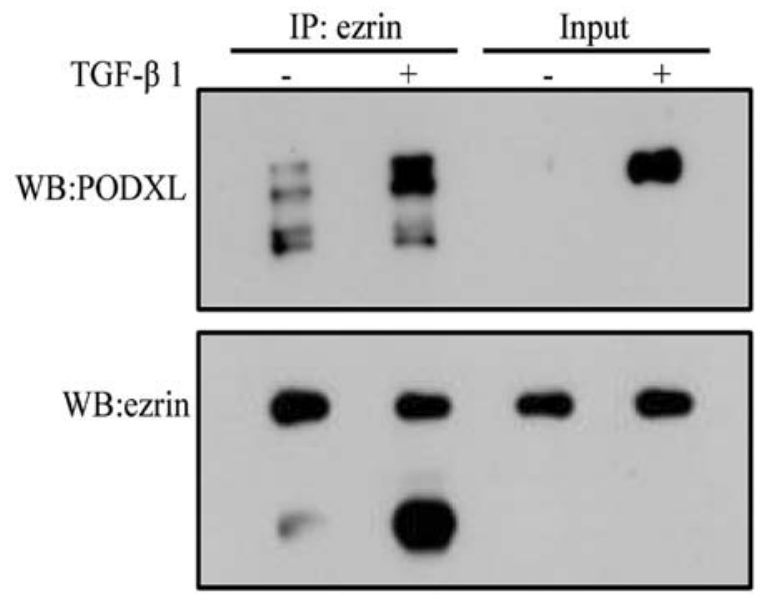

Figure 6. PODXL is associated with ezrin after TGF- $\beta 1$ treatment. A549 cells were cultured without or with TGF- $\beta 1$ for $48 \mathrm{~h}$. Cell lysated from A549 cells was immunoprecipitated with anti-ezrin antibody or IgG. The resulting precipitates as well as the cell lysates were subjected to immunoblot analysis with the indicated antibodies.

we demonstrated that ezrin is associated with morphological changes and actin filament remodeling, and regulates cell migration and invasion in TGF- $\beta 1$-induced EMT, suggesting ezrin plays an important role in TGF- $\beta 1$-induced EMT of human alveolar epithelial cells and its involvement is independent on the expression level (Figs. 3 and 4).

Several studies indicated that the localization of ezrin, rather than its expression level, is correlated with its function (34-36). In breast cancer lines, the total ezrin protein level has no difference, but its localization is associated with dedifferentiation and adverse features in invasive breast tumors and cancer cell lines. In salivary acinar cells from Sjögren's syndrome, the structure and organization of microvilli are linked to the localization changes of ezrin. In the present study, we also found that differential ezrin localization, rather than total ezrin protein levels, is associated with EMT characteristics in lung cell lines (Fig. 5). Increasing number of studies have shown ezrin is concentrated in the microvilli of the cell surface playing a normal role under normal physiological condition or before cytokine stimulation, while it could translocate to the cytoplasm after cytokine stimulation (37). In the cytosol, ezrin exists in a monomeric form and is thought to be 'inactive', provoking dysfunction in ezrin-mediated cellular processes (38-40). Following the stimulation, ezrin is phosphorylated, binds to actin and membrane proteins, colocalizes with F-actin, and redistributes to membrane, which is associated with enhanced migration and invasion (41-44). Our observations were very close to these studies (Fig. 3B). Before TGF- $\beta 1$ induction, ezrin mainly distributes in the microvilli 
and the nucleus. After TGF- $\beta 1$ treatment, ezrin translocates to the cytosol, and with the increase of stimulation time, it co-localizes with F-actin and redistributes to cell membrane. These results indicated that ezrin may be involved in the mechanism of TGF- $\beta 1$-induced EMT through the change of its distribution.

Ezrin is known to link membrane proteins and actin cytoskeleton. Membrane proteins include hyaluronate receptor (CD44), sodium-hydrogen exchanger (NHE), and cell adhesion molecules (ICAM-1, -2 and -3) (45-47). After activated with cytokine, membrane proteins could transduce extracellular signals into intracellular signals through the interaction between their extracellular domain and ligands. Ezrin also unmasks membrane protein and F-actin binding sites. The $\mathrm{N}$-terminal FERM domain of ezrin can bind to the cytoplasmic tail of membrane proteins and its $\mathrm{C}$-terminal domain interacts with F-actin, resulting in actin filament remodeling and provoking migration and invasion (17). We hypothesized that membrane proteins interacting with ezrin may be different with the distribution change of ezrin, initiating a regulatory mechanism of TGF- $\beta 1$-induced EMT. PODXL has been found to participate in cytoskeleton rearrangement and tumor metastasis through interaction with ezrin. PODXL could activate RhoA and induces actin reorganization through NHERF1 and ezrin in MDCK cells (48). It also increases the aggressive phenotype of breast and prostate cancer cells in vitro through its interaction with ezrin (25). In our study, we indicated that PODXL significantly interacts with ezrin with the increased expression of PODXL after TGF- $\beta 1$ treatment (Fig. 6). The result suggests PODXL may be involved in regulating TGF- $\beta 1-$ induced EMT through interaction with ezrin, and ezrin may act as a linker between PODXL and actin cytoskeleton during TGF- $\beta 1$-induced EMT.

In conclusion, we demonstrated that ezrin is associated with actin filament reorganization and cell metastasis during TGF- $\beta 1$-induced alveolar EMT, indicating that ezrin is required for EMT induced by TGF- $\beta 1$. The interaction of ezrin and PODXL may be important in regulating TGF- $\beta 1$-induced EMT.

\section{Acknowledgements}

This study was supported by National Program on Key Basic Research Project (973 Program) (grant no. 2011CB910700), High-Level Talents Project of the Universities of Guangdong (no. [2011]431), National Natural Science Foundation of China (Grant no. 31000628), Fundamental Research Funds for the Central Universities (grant nos. 21611430, 21610101 and 21609317), and Natural Science Foundation of Guangdong Province (grant no. S2013030013315).

\section{References}

1. Lim $\mathbf{J}$ and Thiery JP: Epithelial-mesenchymal transitions: insights from development. Development 139: 3471-3486, 2012.

2. Willis BC and Borok Z: TGF-beta-induced EMT: mechanisms and implications for fibrotic lung disease. Am J Physiol Lung Cell Mol Physiol 293: 525-534, 2007.

3. Savagner P: The epithelial-mesenchymal transition (EMT) phenomenon. Ann Oncol 21 (Suppl 7): vii89-vii92, doi:10.1093/ annonc/mdq292, 2010.

4. Xu J, Lamouille S and Derynck R: TGF-beta-induced epithelial to mesenchymal transition. Cell Res 19: 156-172, 2009.
5. Yilmaz $\mathrm{M}$ and Christofori G: EMT, the cytoskeleton, and cancer cell invasion. Cancer Metastasis Rev 28: 15-33, 2009.

6. Thiery JP, Acloque H, Huang RY and Nieto MA: Epithelialmesenchymal transitions in development and disease. Cell 139: 871-890, 2009.

7. Li H, Wang H, Wang F, Gu Q and Xu X: Snail involves in the transforming growth factor $\beta 1$-mediated epithelial-mesenchymal transition of retinal pigment epithelial cells. PLoS One 6: e23322, 2011.

8. Margetts PJ: Twist: a new player in the epithelial-mesenchymal transition of the peritoneal mesothelial cells. Nephrol Dial Transplant 27: 3978-3981, 2012.

9. Onder TT, Gupta PB, Mani SA, Yang J, Lander ES and Weinberg RA: Loss of E-cadherin promotes metastasis via multiple downstream transcriptional pathways. Cancer Res 68: 3645-3654, 2008

10. Luo W, Fang W, Li S and Yao K: Aberrant expression of nuclear vimentin and related epithelial-mesenchymal transition markers in nasopharyngeal carcinoma. Int J Cancer 131: 1863-1873, 2012.

11. Pagan R, Sánchez A, Martin I, Llobera M, Fabregat I and Vilaró S: Effects of growth and differentiation factors on the epithelial-mesenchymal transition in cultured neonatal rat hepatocytes. J Hepatol 31: 895-904, 1999.

12. Saitoh M and Miyazawa K: Transcirptional and post-transcriptional regulation in TGF- $\beta$-mediated epithelial-mesenchymal transition. J Biochem 151: 563-571, 2012.

13. American Thoracic Society, European Respiration Society: American Thoracic Society/European Respiration Society international multidisciplinary consensus classification of the idiopathic interstitial pneumonias. Am J Respir Crit Care Med 165: 277-304, 2002.

14. Selman M and Pardo A: Alveolar epithelial cell disintegrity and subsequent activation: a key process in pulmonary fibrosis. Am J Respir Crit Care Med 186: 119-121, 2012.

15. Marmai C, Sutheriand RE, Kim KK, et al: Alveolar epithelia cells express mesenchymal proteins in patients with idiopathic pulmonary fibrosis. Am J Physiol Lung Cell Mol Physiol 301: L71-L78, 2011.

16. Kolosova I, Nethery D and Kern JA: Role of Smad2/3 and p38 MAP kinase in TGF- $\beta 1$-induced epithelial-mesenchymal transition of pulmonary epithelial cells. J Cell Physiol 226 $1248-1254,2011$

17. Tsukita S and Yonemura S: Cortical actin organization: lessons from ERM (ezrin/radixin/moesin) proteins. J Biol Chem 274: 34507-34510, 1999

18. Tsukita S, Oishi K, Sato N, Sagara J, Kawai A and Tsukita S ERM family members as molecular linkers between the cell surface glycoprotein CD44 and actin-based cytoskeletons. J Cell Biol 126: 391-401, 1994

19. Buckley ST, Medina C, Kasper M and Ehrhardt C: Interplay between RAGE, CD44, and focal adhesion molecules in epithelial-mesenchymal transition of alveolar epithelial cells. Am J Physiol Lung Cell Mol Physiol 300: L548-L559, 2011.

20. Ehrhardt C, Medina C and Buckley ST: Interaction of RAGE with focal adhesion molecules during alveolar epithelial-mesenchymal transition. FASEB J 25: 865, 2011.

21. Haynes J, Srivastava J, Madson N, Wittmann T and Barber DL: Dynamic actin remodeling during epithelial-mesenchymal transition depends on increased moesin expression. Mol Biol Cell 22: 4750-4764, 2011.

22. Nielsen JS and McNagny KM: The role of podocalyxin in health and disease. J Am Soc Nephrol 20: 1669-1676, 2009.

23. Orlando RA, Takeda T, Zak B, et al: The glomerular epithelial cell anti-adhesin podocalyxin associates with the actin cytoskeleton through interaction with ezrin. J Am Soc Nephrol 12: 1589-1598, 2001

24. Li Y, Li J, Straight SW and Kershaw DB: PDZ domain-mediated interaction of rabbit podocalyxin and $\mathrm{Na}(+) / \mathrm{H}(+)$ exchange regulatory factor-2. Am J Physiol Renal Physiol 282: 1129-1139, 2002.

25. Sizemore S, Cicek M, Sizemore N, Ng KP and Casey G: Podocalyxin increases the aggressive phenotype of breast and prostate cancer cells in vitro through its interaction with ezrin. Cancer Res 67: 6183-6191, 2007.

26. Meng X, Ezzati P and Wilkins JA: Requirement of podocalyxin in TGF-beta induced epithelial mesenchymal transition. PLoS One 6: e18715,2011.

27. Ohtani K, Sakamoto H, Rutherford T, Chen Z, Satoh K and Naftolin F: Ezrin, a membrane-cytoskeletal linking protein, is involved in the process of invasion of endometrial cancer cells. Cancer Lett 147: 31-38, 1999. 
28. Elliott BE, Meens JA, SenGupta SK, Louvard D and Arpin M The membrane cytoskeletal crosslinker ezrin is required for metastasis of breast carcinoma cells. Breast Cancer Res 7: R365-R373, 2005

29. Osawa H, Smith CA, Ra YS, Kongkham P and Rutka JT: The role of the membrane cytoskeleton cross-linker ezrin in medulloblastoma cells. Neuro Oncol 11: 381-393, 2009.

30. Cant SH and Pitcher JA: G protein-coupled receptor kinase 2-mediated phosphorylation of ezrin is required for $\mathrm{G}$ proteincoupled receptor-dependent reorganization of the actin cytoskeleton. Mol Biol Cell 16: 3088-3099, 2005.

31. Crepaldi T, Gautreau A, Comoglio PM, Louvard D and Arpin M: Ezrin is an effector of hepatocyte growth factor-mediated migration and morphogenesis in epithelial cells. J Cell Biol 138: 423-434, 1997.

32. Huang HY, Li CF, Fang FM, Tsai JW, Li SH, Lee YT and Wei HM: Prognostic implication of ezrin overexpression in myxofibrosarcomas. Ann Surg Oncol 17: 3212-3219, 2010.

33. Li Q, Gao H, Xu H, et al: Expression of ezrin correlates with malignant phenotype of lung cancer, and in vitro knockdown of ezrin reverses the aggressive biological behavior of lung cancer cells. Tumour Biol 33: 1493-1504, 2012.

34. Sarrió D, Rodríguez-Pinilla SM, Dotor A, Calero F, Hardisson D and Palacios J: Abnormal ezrin localization is associated with clinicopathological features in invasive breast carcinomas. Breast Cancer Res Treat 98: 71-79, 2006.

35. Pérez P, Aguilera S, Olea N, et al: Aberrant localization of ezrin correlates with salivary acini disorganization in Sjögren's Syndrome. Rheumatology 49: 915-923, 2010.

36. Arslan AA, Silvera D, Arju R, Giashuddin S, Belitskaya-Levy I, Formenti SC and Schneider RJ: Atypical ezrin localization as a marker of locally advanced breast cancer. Breast Cancer Res Treat 134: 981-988, 2012.

37. Berryman M, Franck Z and Bretscher A: Ezrin is concentrated in the apical microvilli of a wide variety of epithelial cells whereas moesin is found primarily in endothelial cells. J Cell Sci 105: 1025-1043, 1993.

38. Bretscher A, Edwards K and Fehon RG: ERM proteins and merlin: integrators at the cell cortex. Nat Rev Mol Cell Biol 3: $586-599,2002$
39. Moilanen J, Lassus H, Leminen A, Vaheri A, Bützow R and Carpén O: Ezrin immunoreactivity in relation to survival in serous ovarian carcinoma patients. Gynecol Oncol 90: 273-281, 2003.

40. Tokunou M, Niki T, Saitoh Y, Imamura H, Sakamoto M and Hirohashi S: Altered expression of the ERM proteins in lung adenocarcinoma. Lab Invest 80: 1643-1650, 2000.

41. Gautreau A, Louvard D and Arpin M: Morphogenic effects of ezrin require a phosphorylation-induced transition from oligomers to monomers at the plasma membrane. J Cell Biol 150: 193-203, 2000

42. Bretscher A: Rapid phosphorylation and reorganization of ezrin and spectrin accompany morphological changes induced in A-431 cells by epidermal growth factor. J Cell Biol 108: 921-930, 1989.

43. Jiang WG, Hiscox S, Singhrao SK, Puntis MC, Nakamura T, Mansel RE and Hallett MB: Induction of tyrosine phosphorylation and translocation of ezrin by hepatocyte growth factor/ scatter factor. Biochem Biophys Res Commun 217: 1062-1069, 1995.

44. Chen Z, Fadiel A, Feng Y, Ohtani K, Rutherford T and Naftolin F: Ovarian epithelial carcinoma tyrosine phosphorylation, cell proliferation, and ezrin translocation are stimulated by interleukin 1alpha and epidermal growth factor. Cancer 92: 3068-3075, 2001

45. Martin TA, Harrison G, Mansel RE and Jiang WG: The role of the CD44/ezrin complex in cancer metastasis. Crit Rev Oncol Hematol 46: 165-186, 2003.

46. Weinman EJ, Steplock D, Donowitz M and Shenolikar S: NHERF associations with sodium-hydrogen exchanger isoform 3 (NHE3) and ezrin are essential for cAMP-mediated phosphorylation and inhibition of NHE3. Biochemistry 39: 6123-6129, 2000.

47. Heiska L, Alfthan K, Grönholm M, Vilja P, Vaheri A and Carpén O: Association of ezrin with intercellular adhesion molecule-1 and -2 (ICAM-1 and ICAM-2). Regulation by phosphatidylinositol 4, 5-bisphosphate. J Biol Chem 273: 21893-21900, 1998.

48. Schmieder S, Nagai M, Oriando RA, Takeda T and Farquhar MG: Podocalyxin activates RhoA and induces actin reorganization through NHERF1 and ezrin in MDCK cells. J Am Soc Nephrol 15: 2289-2298, 2004. 